\title{
Simultaneous PET and Multispectral 3-Dimensional Fluorescence Optical Tomography Imaging System
}

\author{
Changqing Li, Yongfeng Yang, Gregory S. Mitchell, and Simon R. Cherry \\ Department of Biomedical Engineering, University of California, Davis, Davis, California
}

\begin{abstract}
Integrated PET and 3-dimensional (3D) fluorescence optical tomography (FOT) imaging has unique and attractive features for in vivo molecular imaging applications. We have designed, built, and evaluated a simultaneous PET and 3D FOT system. The design of the FOT system is compatible with many existing smallanimal PET scanners. Methods: The 3D FOT system comprises a novel conical mirror that is used to view the whole-body surface of a mouse with an electron-multiplying charge-coupled device camera when a collimated laser beam is projected on the mouse to stimulate fluorescence. The diffusion equation was used to model the propagation of optical photons inside the mouse body, and 3D fluorescence images were reconstructed iteratively from the fluorescence intensity measurements measured from the surface of the mouse. Insertion of the conical mirror into the gantry of a small-animal PET scanner allowed simultaneous PET and 3D FOT imaging. Results: The mutual interactions between PET and 3D FOT were evaluated experimentally. PET has negligible effects on 3D FOT performance. The inserted conical mirror introduces a reduction in the sensitivity and noise-equivalent count rate of the PET system and increases the scatter fraction. PET-FOT phantom experiments were performed. An in vivo experiment using both PET and FOT was also performed. Conclusion: Phantom and in vivo experiments demonstrate the feasibility of simultaneous PET and 3D FOT imaging. The first in vivo simultaneous PET-FOT results are reported.
\end{abstract}

Key Words: fluorescence; optical imaging; PET; small-animal imaging

J Nucl Med 2011; 52:1268-1275

DOI: 10.2967/jnumed.110.082859

\section{A}

perfect biomedical imaging modality would provide anatomic, functional, physiologic, and molecular information. The absence of such a modality is a driving force for multimodality imaging development, especially the combination of high-spatial-resolution imaging modalities such as CT and MRI with high-sensitivity molecular imaging modalities such as PET and optical imaging (1-3). However, there also are strong scientific arguments for having

Received Aug. 31, 2010; revision accepted Mar. 29, 2011.

For correspondence or reprints contact: Changqing Li, Department of Biomedical Engineering, University of California, Davis, 451 Health Sciences Dr., Davis, CA 95616.

E-mail: cqli@ucdavis.edu

COPYRIGHT @ 2011 by the Society of Nuclear Medicine, Inc. the ability to perform 2 functional imaging modalities (3dimensional [3D] fluorescence optical tomography [FOT] and PET) simultaneously (4).

First, several laboratories are developing multimodality reporter systems for imaging gene expression or targeted imaging probes or drug delivery vehicles in which a molecule or particle is labeled with both a PET radionuclide and a fluorophore (5-8). A simultaneous PET and FOT instrument would allow concurrent characterization of the temporal and spatial characteristics of these new molecular imaging probes with PET, offering quantitative validation of the biodistribution and pharmacokinetics of the probe.

Second, PET for small-animal imaging is a well-developed technology, with spatial resolution around $1 \mathrm{~mm}$ (9). Although 3D FOT for small-animal imaging is being studied by many groups, with promising results $(10-13)$, it is still being developed and refined. PET with dual-labeled probes or particles can be used to evaluate and validate data collection and image reconstruction strategies used for 3D FOT imaging. A combined PET-FOT system therefore can help the development of quantitative 3D FOT techniques and methods.

Third, these 2 molecular imaging modalities may provide complementary information. For imaging tumor models in small animals, PET with ${ }^{18} \mathrm{~F}$-FDG measures metabolic activity of the tumor, whereas FOT imaging with an enzymeactivatable probe can measure tumor protease activity (14). For a drug delivery study, FOT can monitor the tumor response to a drug (e.g., expression level of the gene or protein targeted by the drug using fluorescent reporters), whereas PET is able to quantify the distribution of the drug inside the subject. Another key application is in quantifying signals from activatable fluorescence probes. If these probes are dual-labeled, PET will provide a signal proportional to the total amount of probe present in a tissue of interest, and FOT will provide a signal proportional to the amount of probe that is activated at that location. Both pieces of information are required to quantify the fraction of the probe that has been modified by its interaction with the target protein of interest.

Finally, PET data and FOT data can be used synergistically if there is some level of correlation expected between spatial and temporal distribution of the probe in the PET and FOT images $(4,15)$. This could, for example, result in reconstructed FOT images with a resolution comparable to that obtained with PET $(\sim 1 \mathrm{~mm})$. 
Overall, the goal of a combined PET-FOT instrument is to overcome some of the inherent limitations in both modalities by providing spatially and temporally synchronized data from identical dual-labeled or complementary but separately radio- and fluorescently labeled probes that can improve analysis or quantification of biologic processes in healthy tissue, for disease states, and in response to therapies. Modern preclinical PET scanners can reach a spatial resolution of approximately $1 \mathrm{~mm}$ and can detect radiotracers present in nano- to picomolar concentrations. Compared with optical imaging, the signal is much less depth-dependent, and correction for photon attenuation and scatter is quite accurate. However, a disadvantage of PET radiotracers is that they are always on and so cannot be switched by biologic events. Furthermore, there are no direct PET reporter proteins (nothing analogous to green fluorescent protein and other fluorescent reporters); rather, gene expression must be imaged indirectly using a reporter protein that can sequester or bind a PET radiotracer (16). FOT also can detect low concentrations of fluorescent molecules, but the signal and spatial resolution achievable are extremely sensitive to depth and tissue optical properties. Typical reconstructed resolution for a target deep inside the body can be many millimeters. On the other hand, fluorescent probes can be designed to be activated on encountering a target or have characteristics that depend on biologically important parameters such as $\mathrm{pH}$ or calcium concentration. The careful design of experiments with such probes in a combined PET-FOT system has the potential to improve quantification and understanding of the underlying biologic targets through the complementary information that is provided.

Prompted by this motivation, several prototype systems combining PET or SPECT with optical imaging for smallanimal studies have been developed. Peter et al. have reported a microlens array-based optical PET system. This imaging system has been evaluated with phantom experiments but was found to have lower sensitivity than a charge-coupled device (CCD)-based optical imaging system (17). Jung et al. have developed a dual-modality imaging system combining a $\gamma$-camera and optical imager for small animals with position-sensitive photomultiplier tubes for detecting both $\gamma$-ray photons and optical photons. Initial phantom experiments were reported (18). Alexandrakis et al. have proposed a concept for an optical PET system that uses a common detector for detecting the signal from both modalities and simulated it with numeric studies (19).

In this paper, we describe our simultaneous 3D FOT and PET system for small-animal imaging. The FOT system is based on a conical mirror geometry that is readily inserted into conventional small-animal PET scanners with a cylindric geometry. Although each of the approaches described has its unique features and strengths, the system we have designed and evaluated has the following advantages. First, the added cost of inserting a mirror into an existing PET scanner is low. The proposed method can work in many preclinical PET scanners in which there is sufficient bore to insert a conical mirror. The approach is thus broadly applicable as an add-on to an existing PET system. Second, optical imaging sensitivity is high, because standard high-sensitivity CCD cameras can be used and there is no reason for any degradation in the performance of the FOT system when operated inside the PET system. Third, we have the flexibility to select different fluorescence illumination patterns to maximize the information available for FOT reconstruction. Fourth, PET and 3D FOT image fusion and joint reconstruction will benefit from the fact that the PET scanner, conical mirror, CCD camera, and imaged mouse are all rigidly fixed in 1 place for the entire data acquisition procedure.

The stand-alone 3D FOT imaging system has been reported in detail elsewhere (20), and we have also previously proposed FOT reconstruction algorithms that use the PET reconstruction as prior information (15). In this paper, we reconfigure the 3D FOT system to make it compatible with our existing microPET II small-animal PET scanner (21) and integrate the 2 systems to enable simultaneous PET and FOT imaging. In these experiments, FOT data are reconstructed without using any guidance or prior information from PET. We focus on measuring the effects of 3D FOT and PET systems on each other after the conical mirror has been inserted into the PET scanner. Both phantom and in vivo experiments are performed to evaluate this dualmodality imaging system.

\section{MATERIALS AND METHODS}

\section{D FOT Imaging System}

The 3D FOT system uses a truncated conical mirror allowing simultaneous viewing of the entire surface of the animal by an electron-multiplying CCD (EMCCD) camera. This geometry and the small size of the conical mirror (outside diameter, $153 \mathrm{~mm}$; total length, $62.5 \mathrm{~mm}$; apex angle, $90^{\circ}$ ) make it feasible to insert the conical mirror inside the gantry of our existing microPET II scanner for simultaneous PET and 3D FOT imaging. Figure 1A shows the design of the conical mirror, which was made of aluminum and coated with silver. The outside diameter is $1 \mathrm{~mm}$ smaller than the bore of the microPET II and therefore fits snugly inside. The thickness of the aluminum structure is $7 \mathrm{~mm}$, which provides rigidity and prevents any warping that would lead to image distortions.

Figure 1A shows a schematic of the 3D FOT imaging system. A 650-nm laser (BWF-OEM-650-200; B\&W Tech Inc.) with a collimating lens at the end of a pigtail fiber was used to excite the fluorescence dye, and then a bandpass filter (650FS20-50 [650-nm central wavelength, with 20-nm bandwidth]; Andover Corp.) was used to remove light at other wavelengths. Another lens focused the beam into a small spot of 1-mm diameter at the mouse surface, and 2 motorized mirrors scanned the laser beam across the subject. A glass bed was placed in the center of the conical mirror. Bandpass filters, placed inside a filter wheel, were used to select emission wavelengths. In the front of the filter wheel, a long-pass filter (665FG07-50 [Andover Corp.] for phantom experiment; LP02785RU-25 [Semrock Inc.] for in vivo experiment) was used to remove excitation laser spillover into the measurements at the emission wavelengths. An EMCCD (C9100-3; Hamamatsu Corp.) was used to collect the emitted fluorescence photons. 


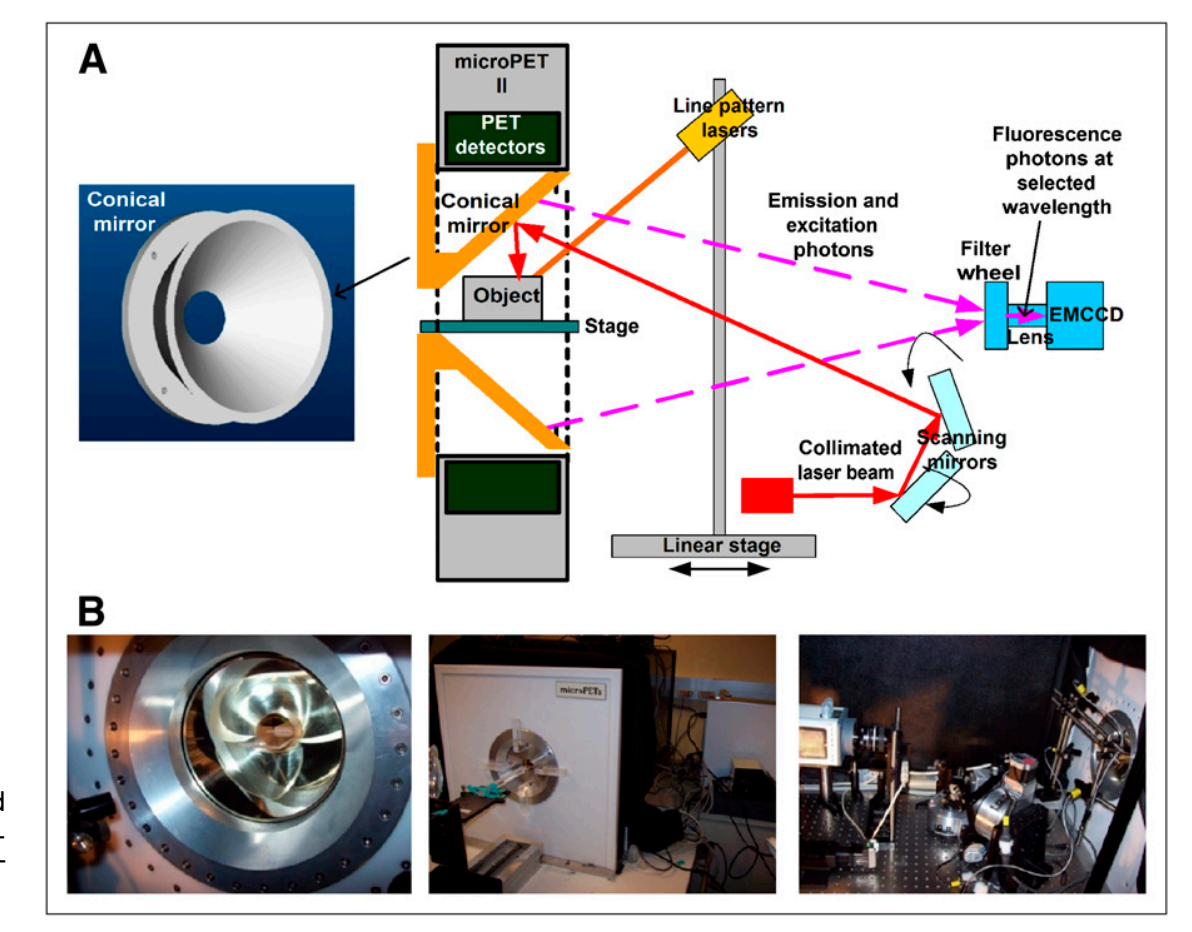

FIGURE 1. (A) Design of conical mirror and schematic of 3D FOT system. (B) Photographs of integration of $3 \mathrm{D}$ FOT and PET systems.

Three line pattern lasers, mounted on a linear stage, were used to project lines onto the top and side surfaces of the mouse and were scanned axially along the mouse to extract the surface geometry. The bottom surface was assumed to be a flat plane based on the geometry of the imaging platform on which the object or animal was placed. To minimize ambient light effects on FOT imaging, the open aperture of the conical mirror and the gap between the FOT enclosure and PET gantry were covered with black cloth. The performance of the 3D FOT system and the algorithms used for surface profiling and 3D FOT reconstruction have been reported previously (20).

\section{microPET II Scanner}

The microPET II scanner and its performance have been described in detail elsewhere $(21,22)$. Briefly, a microPET II detector module consists of an array of $14 \times 14$ lutetium oxyorthosilicate crystals, each of which has a cross section of $0.975 \times$ $0.975 \mathrm{~mm}$ and a length of $12.5 \mathrm{~mm}$. The scanner consists of 3 rings of detector modules, with 30 detector modules per ring. The axial field of view is $4.9 \mathrm{~cm}$, and the transaxial field of view is $8.5 \mathrm{~cm}$. The aperture of the scanner has a diameter of $15.3 \mathrm{~cm}$.

For simultaneous PET and FOT, the conical mirror was inserted inside the aperture encompassing the entire axial field of view of the microPET II scanner (Fig. 1). The remaining components of the optical imaging system were placed inside a light-tight black box on an optical table at the rear of the microPET II scanner (Fig. 1B). One computer was used to control the EMCCD and the motors in the fluorescence optical imaging system. Another computer, with modified microPET system software (Siemens Preclinical Solutions), was used to control the microPET II scanner for PET data acquisition.

\section{Effect of FOT System on PET Performance}

The conical mirror is made of 7-mm-thick aluminum and is coated with silver several micrometers in thickness. To investigate the effects of the conical mirror on $511-\mathrm{keV}$ photon scattering and attenuation, a point source $\left({ }^{68} \mathrm{Ge}, 660 \mathrm{kBq}\right)$ mounted on a linear translation stage was placed at the center of the transaxial field of view of the microPET II scanner, and data were acquired with and without the conical mirror present. Axial variations are expected because of the conical shape of the mirror and the supporting structure at one end (Fig. 1A). Measurements were acquired in 3-mm steps along the axial field of view of the scanner. For each axial position, data were acquired for $60 \mathrm{~s}$ with a fixed energy window (150-750 keV) and a coincidence timing window of $10 \mathrm{~ns}$. These are the default values used for mouse imaging with this scanner (22). At each axial position, the prompt and random coincident counts were measured simultaneously. The true counts were obtained by subtracting the random counts from the prompt counts. The sensitivity was calculated as $(1-S F) \times \mathrm{R}$, where $S F$ is the scatter fraction at each position and $\mathrm{R}$ is the ratio of true counts to the known number of positron-emitting decays from the ${ }^{68} \mathrm{Ge}$ during the measurement period. The branching ratio for $\beta^{+}$ emission for ${ }^{68} \mathrm{Ga}$ (daughter nuclide of ${ }^{68} \mathrm{Ge}$ ) was accounted for, and secular equilibrium between the parent and daughter nuclide was assumed.

The scatter fraction from the mirror and scanner gantry was measured at each location as follows. List-mode data were sorted by single-slice rebinning into sinograms, with a maximum ring difference of 41 , and random counts were subtracted. The sinograms were summed across all projection angles and slices to create a single-slice profile. The scattered events were taken to be the summed coincident events at transverse positions more than $5 \mathrm{~mm}$ away from the center. The scatter fraction was defined as the ratio of the scattered events to the total events.

A mouse-sized phantom, filled with $33 \mathrm{~cm}^{3}$ of ${ }^{18} \mathrm{~F}-\mathrm{FDG}$ solution with an initial activity of $215 \mathrm{MBq}$, was used to evaluate the noise-equivalent counting (NEC) rate performance of the microPET II scanner with and without the conical mirror. The phantom was fixed on a thin glass platform in the center of the field of view of the microPET II scanner. With the conical mirror inserted, data 
were acquired for $1 \mathrm{~min}$. Then the conical mirror was removed, without disturbing the phantom, and another set of data were acquired for $1 \mathrm{~min}$. The measurements were repeated every $30 \mathrm{~min}$ for $12 \mathrm{~h}$. The energy window was $150-750 \mathrm{keV}$, and the coincidence timing window was $10 \mathrm{~ns}$. Prompt $(P)$ and random $(R)$ counting rates were recorded for each measurement. True $(T)$, scatter $(S)$, and NEC rates were calculated using the following equations,

$$
\begin{gathered}
T=(P-R) \times(1-S F) \\
S=(P-R) \times S F \\
\mathrm{NEC}=\frac{T^{2}}{T+S+2 K R},
\end{gathered}
$$

where $S F$ is the scatter fraction at each position calculated in the section above. $K$ is the fraction of the field of view occupied by the mouse-sized phantom and had a value of 0.294 in this study.

To evaluate any effect of the conical mirror on reconstructed spatial resolution, a cold cylindric phantom (diameter, $2.5 \mathrm{~cm}$; length, $4 \mathrm{~cm}$ ) containing 3 line sources was placed at the center of the field of view of the scanner. The line sources were made from steel needles (inner diameter, $0.1 \mathrm{~mm}$; outer diameter, $0.2 \mathrm{~mm}$ ) and were filled with high-concentration ${ }^{18} \mathrm{~F}$-FDG solution. The needles were located at radial offsets of $0,7.5$, and $15 \mathrm{~mm}$. Data were acquired for 10 min with an energy window from 150 to 750 $\mathrm{keV}$ and a $10-\mathrm{ns}$ coincidence timing window. The 3D sinogram set was Fourier-rebinned, and conventional 2-dimensional filtered backprojection with a ramp filter (cutoff at Nyquist frequency) was used to reconstruct the images. To satisfy sampling criteria for determining the spatial resolution, and to accommodate all 3 line sources in the field of view, a matrix size of $512 \times 512$ pixels and a pixel size of $0.168 \mathrm{~mm}$ was used for the reconstruction of each slice.

\section{Effect of PET on 3D FOT Performance}

There are 2 constraints or effects of integrating the FOT system with the microPET II system. First, the microPET II bore size places limits on the outer diameter of the conical mirror, limiting the axial field of view. Currently, the mirror has an axial field of view of $6.25 \mathrm{~cm}$, slightly shorter than the length of a typical mouse but larger, nonetheless, than the axial field of view of this particular PET scanner $(4.9 \mathrm{~cm})$. Second, there is the potential for 511-keV photons emitted within the subject to directly interact in the CCD camera, possibly leading to saturated pixels. To test effects of radioactivity in the subject on CCD performance, a cylindric mouse-sized phantom (volume, $33 \mathrm{~cm}^{3}$ ) was filled with an initial activity of $54 \mathrm{MBq}$ of ${ }^{18} \mathrm{~F}$ and placed inside the center of the field of view of the system. The EMCCD camera took 10 images (exposure time, $10 \mathrm{~s}$ each) every $30 \mathrm{~min}$ as the activity decayed. The distance from the phantom to the CCD camera in the system was $647.7 \mathrm{~mm}$.

The number of direct $511-\mathrm{keV}$ photon interactions in the EMCCD is easily determined by counting the number of bright pixels, as a 511-keV photon interaction leads to a high signal in that CCD pixel. A small number of these interactions may also be due to cosmic rays. Many CCD cameras have built-in corrections to eliminate signal in pixels because of high-energy interactions from cosmic rays; no such correction was used in the data collected here.

\section{Phantom Experiment}

A $32 \times 32 \times 29 \mathrm{~mm}$ cubic phantom, composed of $1 \%$ intralipid and $2 \%$ agar (20) to mimic the scattering properties of tissue, was prepared. The phantom had 4 embedded capillary tube targets with an inner diameter of $1 \mathrm{~mm}$, outside diameter of $1.5 \mathrm{~mm}$, and length of $12 \mathrm{~mm}$. Two of the capillary tubes were filled with both ${ }^{18} \mathrm{~F}-\mathrm{FDG}$ solution $(3.7 \mathrm{MBq}$ ) and $4.6 \mu \mathrm{M} \mathrm{DiD}$ solution (excitation peak, $650 \mathrm{~nm}$, and emission peak, $670 \mathrm{~nm}\left[1,1^{\prime}\right.$-dioctadecyl-3,3,3', $3^{\prime}$ tetramethylindodicarbocyanine perchlorate, D307; Invitrogen]). The other 2 were filled with ${ }^{18}$ F-FDG solution $(3.7 \mathrm{MBq})$ only. Figure 2 shows the phantom geometry and the 4 embedded targets. microPET II and optical fluorescence data were taken simultaneously.

For PET, data were collected with an energy window of 150-750 $\mathrm{keV}$ and a 10-ns coincidence timing window. Data were reconstructed using filtered backprojection with a ramp filter cutoff at the Nyquist frequency (matrix size, $512 \times 512 \times 83$; transverse pixel size, $0.168 \mathrm{~mm}$; and slice thickness, $0.58 \mathrm{~mm}$ ). For FOT, the collimated excitation laser illuminated 20 positions on the front surface (the surface closest to the EMCCD), and a fluorescence image was obtained at $720 \mathrm{~nm}$ for each excitation position.

For FOT reconstruction, a finite element mesh with 8,690 nodes and 47,581 tetrahedral elements was used for optical forward model calculation based on the diffusion equation (20). Detector nodes $(1,057)$ were selected on the 4 side surfaces of the phantom. The system matrix was solved iteratively by a preconditioned conjugate gradient method. The image reconstruction process corrects for the geometric distortion introduced by the conical mirror. Light emission from the surface of the object is assumed to be Lambertian. On the basis of the optics and geometry of the FOT system, each CCD pixel subtends approximately the same solid angle to the object surface; therefore, detailed modeling of light propagation from the surface to the CCD camera was not performed. The total data acquisition time was approximately $30 \mathrm{~min}$ for both modalities.

\section{In Vivo Experiment}

As proof of concept for in vivo hybrid PET-FOT imaging, a 30-g nude mouse with an implanted melanoma (DX-6) xenograft (right side, dorsal) was anesthetized (2\% isoflurane) and placed in the center of the field of view of the dual-modality system. The tumor measured approximately $7 \mathrm{~mm}$ across. The mouse was injected intravenously with $10 \mathrm{nmol} 2$-deoxyglucose (2-DG) conjugated with IRDye $800 \mathrm{CW}$ (23) (LICOR) $24 \mathrm{~h}$ before imaging and $5 \mathrm{MBq}$ of ${ }^{18} \mathrm{~F}-\mathrm{FDG} 50 \mathrm{~min}$ before imaging. The precise mechanism by which fluorescent 2-DG accumulates in cells is still under investigation. There is strong evidence that accumulation depends on binding to glucose transporters, that uptake can be blocked in a competitive binding assay with glucose, and that

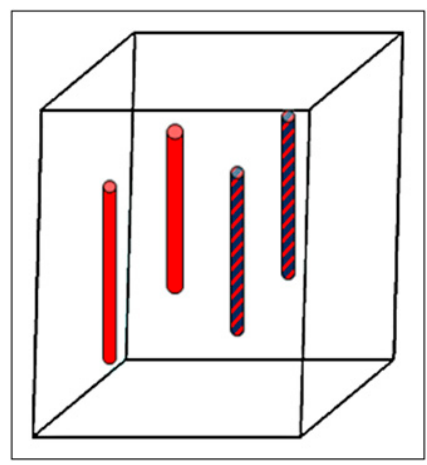

FIGURE 2. Schematic of phantom geometry and location of 4 targets. Patterned bars indicate both $18 \mathrm{~F}-\mathrm{FDG}$ and DiD solution, and plain red bars indicate ${ }^{18} \mathrm{~F}-\mathrm{FDG}$ solution only. 
enhanced uptake is seen in many tumor cell lines and in xenograft models (23). The kinetics of this probe (both uptake and clearance), however, are far slower than ${ }^{18} \mathrm{~F}-\mathrm{FDG}$, with optimal imaging times being approximately $24 \mathrm{~h}$ after injection. The IRDye 800 CW conjugated with 2-DG has an excitation peak wavelength of $774 \mathrm{~nm}$ and emission peak wavelength of $791 \mathrm{~nm}$.

First, the subject was scanned by translating the 3 orthogonal line lasers along the length of the mouse to generate the mouse surface geometry. Figure 3 (top) plots the extracted surface points that were used to generated a 3D finite element mesh with 16,126 nodes and 85,533 tetrahedral elements (Fig. 3, bottom). Then, the collimated excitation laser $(785 \mathrm{~nm})$ illuminated the mouse surface at 55 positions (spot size, $\sim 1 \mathrm{~mm}$ diameter) covering 4 sides of the mouse. For each excitation position, a fluorescence image was obtained at $820 \mathrm{~nm}$ (filter bandwidth, $12 \mathrm{~nm}$ ). The EMCCD exposure times are between 1 and $5 \mathrm{~s}$, depending on the laser excitation position. At the same time, the microPET II scanner acquired PET data (single bed position, static scan) with an energy window of 150-750 keV and coincidence timing window of $10 \mathrm{~ns}$. Dead time and randoms were negligible. The simultaneous PET and optical data acquisition lasted $30 \mathrm{~min}$. Next, the mouse, while still under anesthesia and on the same imaging stage, was moved into a micro-CT scanner (Inveon SPECT/CT; Siemens Preclinical Solutions) for imaging ( $80 \mathrm{kVp}, 0.2 \mathrm{~mA}, 180$ projections). Finally, the mouse was euthanized, frozen, and sectioned at different transverse levels. Each cryosection was imaged by an IVIS 100 system (Caliper Life Sciences) using the available ICG filter (bandpass filter from 810 to $875 \mathrm{~nm}$ ) to measure fluorescence in that section.
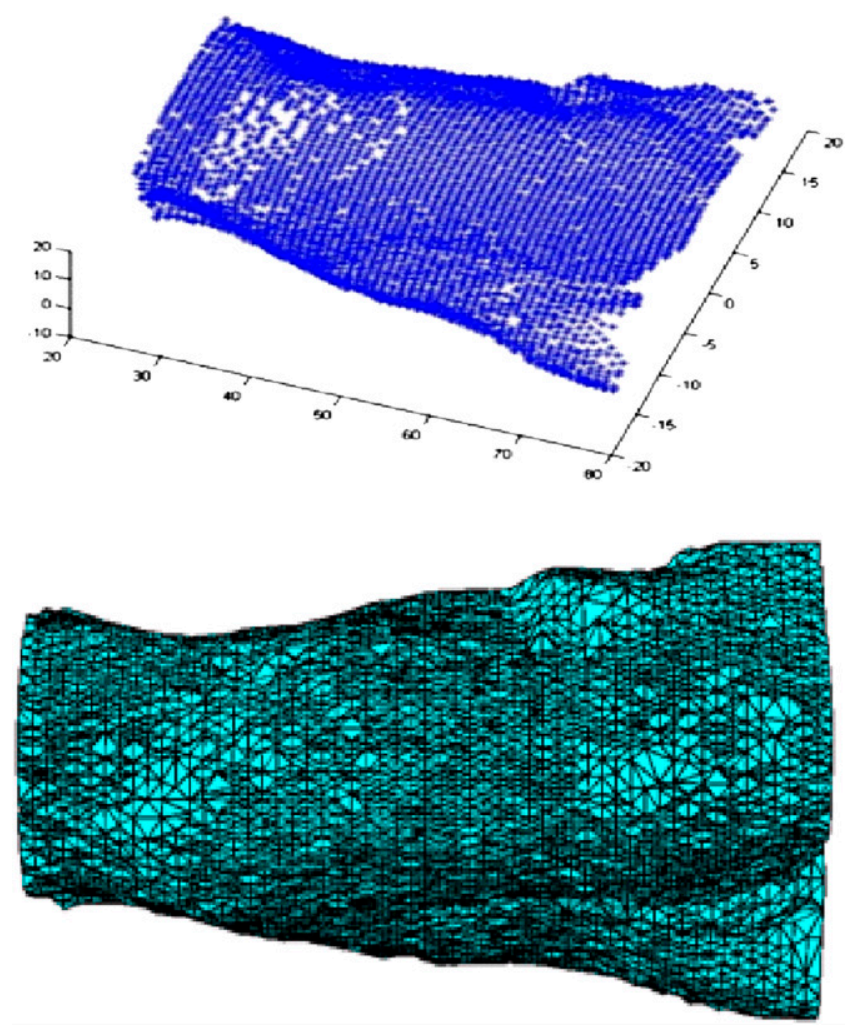

FIGURE 3. Extracted mouse surface geometry obtained by scanning 3 line pattern lasers along length of mouse (top); 3D finite element mesh for optical image reconstruction (bottom).
For 3D FOT image reconstruction, the diffusion equation, modeling photon propagation inside the mouse with the finite element method, was used to obtain the forward system matrix (20). Geometric distortions from the conical mirror were corrected. The optical properties were assumed to be uniform, with an absorption coefficient of $0.022 \mathrm{~mm}^{-1}$ and reduced scattering coefficient of $1.41 \mathrm{~mm}^{-1}$ at both excitation and emission wavelengths. Images of fluorescence dye concentration were then reconstructed with the preconditioned conjugate gradient method. Autofluorescence was ignored, because at the excitation wavelengths used, autofluorescence is low and the measured signal is dominated by the exogenous fluorescent probe. The PET data were reconstructed using a 3D maximum a posteriori method (24). In common with our standard protocol on this scanner, no attenuation or scatter correction was applied to the PET data. This study was conducted under a protocol approved by the University of California, Davis Institutional Animal Care and Use Committee.

\section{RESULTS}

\section{Effect of FOT System on PET Performance}

With the point source at different axial positions in the microPET II gantry, sensitivity and scatter fractions were calculated and are plotted in Figures $4 \mathrm{~A}$ and $4 \mathrm{~B}$. In the center of the field of view, the sensitivity is reduced from $2.4 \%$ to $1.7 \%$, and the scatter fraction is increased from $19 \%$ to $37 \%$ by the presence of the conical mirror. The increase in scatter fraction is not axially symmetric because of the shape of the mirror and mounting flange at 1 end.

The NEC rates were calculated and plotted in Figure 4C with and without the conical mirror. Because of scattering and attenuation from the conical mirror, the NEC with the mirror is reduced by roughly $44 \%-54 \%$ in the activity concentration range typically found in animal PET studies (0.11-0.22 $\left.\mathrm{MBq} / \mathrm{cm}^{3}\right)$.

To calculate the spatial resolution, profiles in the radial direction through the 3 line sources were obtained, and the full width at half maximum was determined. Figure 4D shows the profile for the central line source in the tangential direction, with and without the mirror inserted. Table 1 summarizes the results, which indicate that the mirror degrades spatial resolution by less than $10 \%$ in both radial and tangential directions.

\section{Effect of PET on 3D FOT Performance}

Figure 5 (top) shows a typical EMCCD image, with 3 pixels in which 511-keV photons have directly interacted, and the number of high-energy photons detected by the EMCCD as a function of radionuclide activity (bottom). The roughly linear trend with phantom activity suggests that these direct hits come primarily from 511-keV annihilation photons from the source rather than cosmic or other background sources. The leftmost point on the curve corresponds to an activity of 5.0 $\mathrm{MBq}$, which produces, on average, 0.1 direct $511-\mathrm{keV}$ interactions per second in the EMCCD. For most optical measurements, the EMCCD exposure time is $5 \mathrm{~s}$ or less; thus, for typical radiotracer doses of 3.7-7.4 MBq in a mouse, the rate of direct hits will 


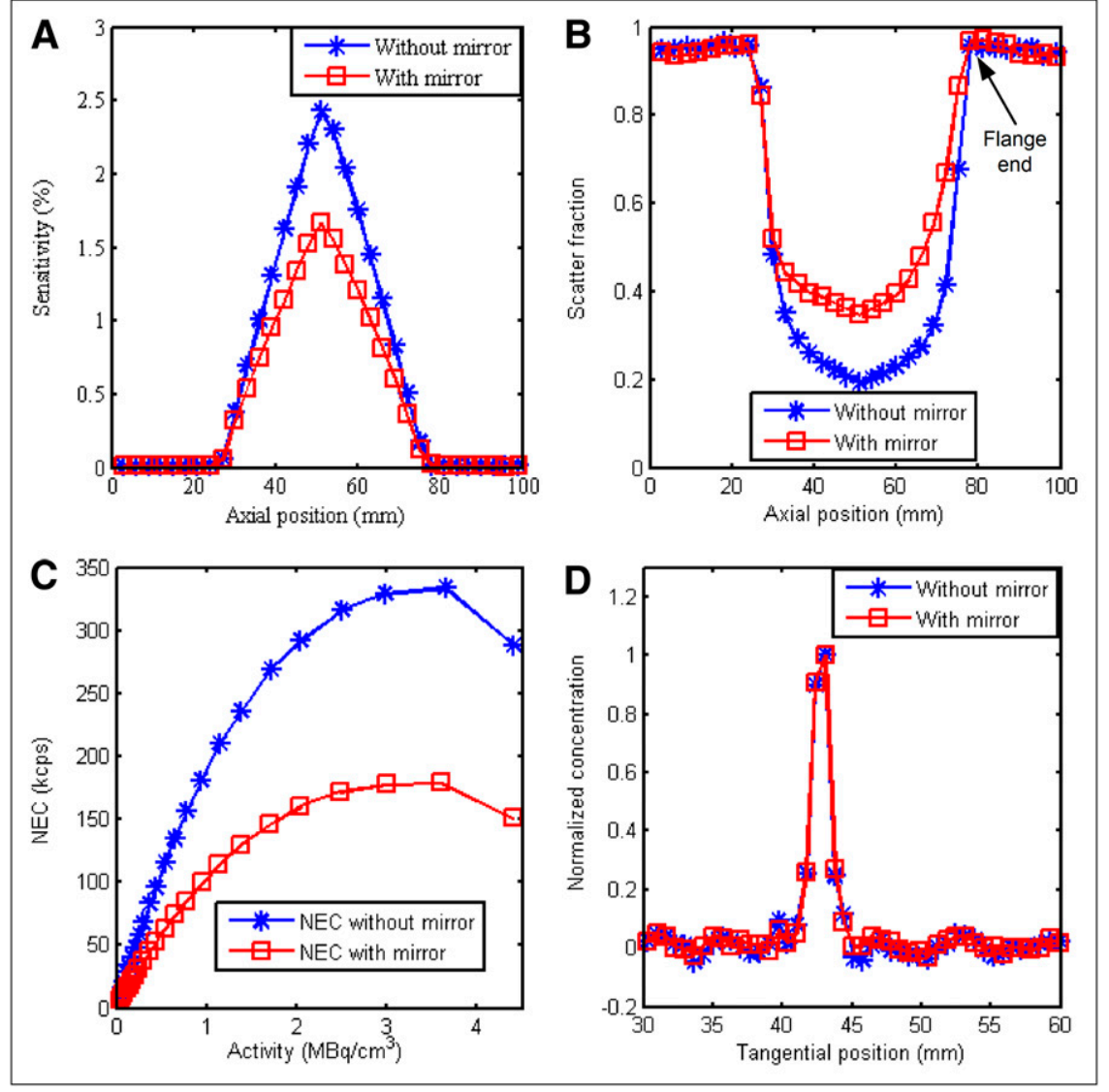

FIGURE 4. PET performance measured with and without inserted mirror: sensitivity using point source (A), scatter fraction using point source (location of flange end of mirror with respect to microPET scanner is indicated (B), NEC using mouse-sized phantom (C), and spatial resolution using line source (D). be less than approximately 0.5 per EMCCD frame. This is therefore a negligible problem.

\section{Phantom Experiment}

For the phantom experiment, the reconstructed 3D FOT images and PET images are fused and shown in Figure 6 in transverse, coronal, and sagittal sections. There is excellent colocalization of the reconstructed ${ }^{18} \mathrm{~F}-\mathrm{FDG}$ and DiD signals in the 2 tubes containing both agents. No signal is seen in the FOT images of the targets that contain only ${ }^{18} \mathrm{~F}-\mathrm{FDG}$.

\section{In Vivo Experiment}

Transverse and coronal slices from the reconstructed ${ }^{18} \mathrm{~F}$ FDG PET and 2-DG FOT images, registered to micro-CT images, are shown in Figure 7A. Fusion with the CT images was accomplished using AMIDE (amide.sourceforge.net), with all image volumes being rescaled to account for differences in voxel size. Datasets were registered manually. At 24 $\mathrm{h}$ after injection, the 2-DG has cleared from most of the body, and signal primarily comes from the region of the tumor and some residual signal in bladder (see transaxial slice). The ${ }^{18} \mathrm{~F}-$ FDG PET image, taken between 50 and 80 min after injection, shows clearance through the kidneys with high bladder signal, high heart uptake (typical with isoflurane anesthesia), and a signal from the tumor that is barely above background. The transverse FOT image across the bladder and tumor is consistent with the cryosection image obtained at roughly the same level (Fig. 7B) and confirms high 2-DG uptake seen in the reconstructed FOT image. 2-DG uptake in the heart was low, as also validated by the cryosection images (not shown). In this particular xenograft model, 2-DG, compared with ${ }^{18} \mathrm{~F}-\mathrm{FDG}$, provides excellent contrast in the tumor.

\section{DISCUSSION}

The aluminum conical mirror results in a significant reduction in sensitivity and increase in scatter fraction, leading to degradation in NEC. Spatial resolution is only

TABLE 1

Spatial Resolution (Full Width at Half Maximum) Measured for 3 Line Sources Along Radial and Tangential Directions, With and Without Mirror

\begin{tabular}{lccc}
\hline Offset $(\mathrm{mm})$ & $\begin{array}{c}\text { With mirror } \\
(\mathrm{mm})\end{array}$ & $\begin{array}{c}\text { Without mirror } \\
(\mathrm{mm})\end{array}$ & $\begin{array}{c}\text { Difference } \\
(\%)\end{array}$ \\
\hline $0 \quad$ Radial & 1.71 & 1.57 & 8.9 \\
$\quad$ Tangential & 1.41 & 1.40 & 0.7 \\
7.5 & & & \\
$\quad$ Radial & 2.03 & 2.11 & -3.7 \\
$\quad$ Tangential & 1.71 & 1.65 & 3.6 \\
$15 \quad$ Radial & 2.11 & 2.02 & 4.4 \\
$\quad$ Tangential & 1.80 & 1.76 & 2.3 \\
\end{tabular}

Offsets for line sources were $0,7.5$, and $15 \mathrm{~mm}$. 


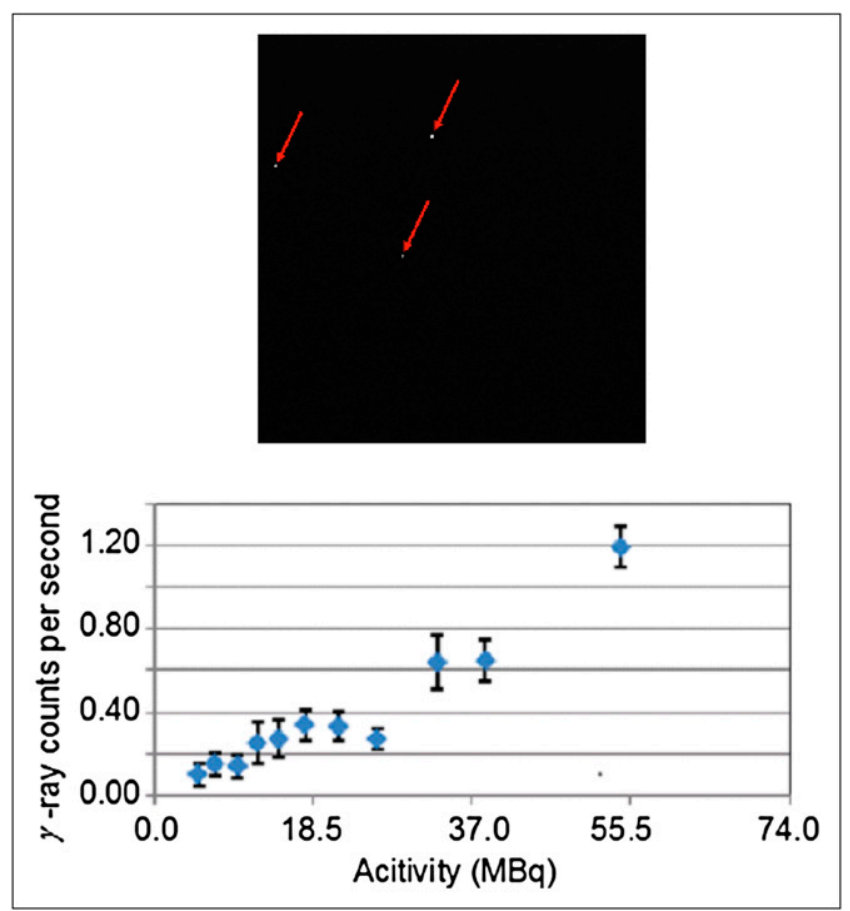

FIGURE 5. Saturated pixels caused by direct 511-keV hits on EMCCD (top); direct $\gamma$-ray hits on EMCCD per second at different activity levels inside mouse-sized phantom (bottom).

slightly degraded by the presence of the mirror. We anticipate that these effects could be significantly reduced using a thinner mirror substrate fabricated from a loweratomic-number material. There was no impact on FOT imaging by integrating the FOT system with the microPET II scanner.

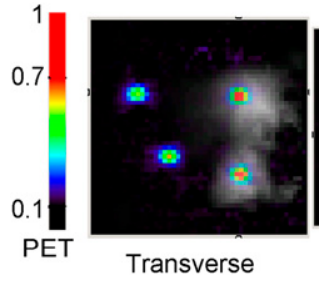

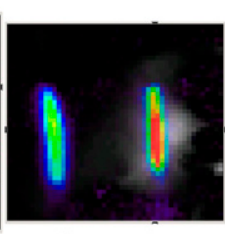

Coronal

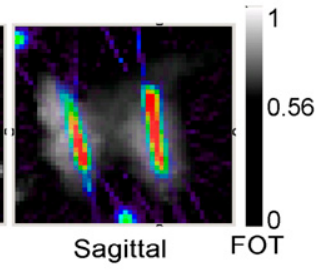

FIGURE 6. Fused reconstructed phantom images. Fluorescence DiD concentration (gray scale) and PET ${ }^{18} \mathrm{~F}-\mathrm{FDG}$ concentration images (color) in transverse, coronal, and sagittal sections. PET and FOT scale bars indicate normalized concentrations with arbitrary units.

In the phantom experiment, the distribution of the radionuclide and fluorophore were accurately reconstructed in terms of location. To further improve localization accuracy, as well as the quantitative accuracy of the FOT system, several enhancements are planned. These include more excitation locations across multiple surfaces of the phantoms, excitation and detection at multiple wavelengths, increased cooling of the EMCCD camera to reduce the noise, and improved rejection of ambient light.

For the in vivo study, these simultaneously acquired datasets show the ability to localize signal in both FOT and PET. This example is challenging for FOT, as it involves a systemically administered, nonactivatable probe, with a broad distribution throughout the body. It thus represents one of the least favorable cases for reconstruction. Although the tumor is fairly superficial, it still has a certain size and depth and therefore FOT has the potential to be of value in improving quantification in this situation.
FIGURE 7. (A) Reconstructed 18F-FDG PET images fused with micro-CT (left). Coronal and transverse slices at level of tumor. Threshold was applied to PET images to show biodistribution despite high activity in bladder. Corresponding slices from 3D FOT reconstruction fused with micro-CT (right). (B) Cryosection image of fluorescence from 2-DG at approximately same level as in vivo transverse slices.

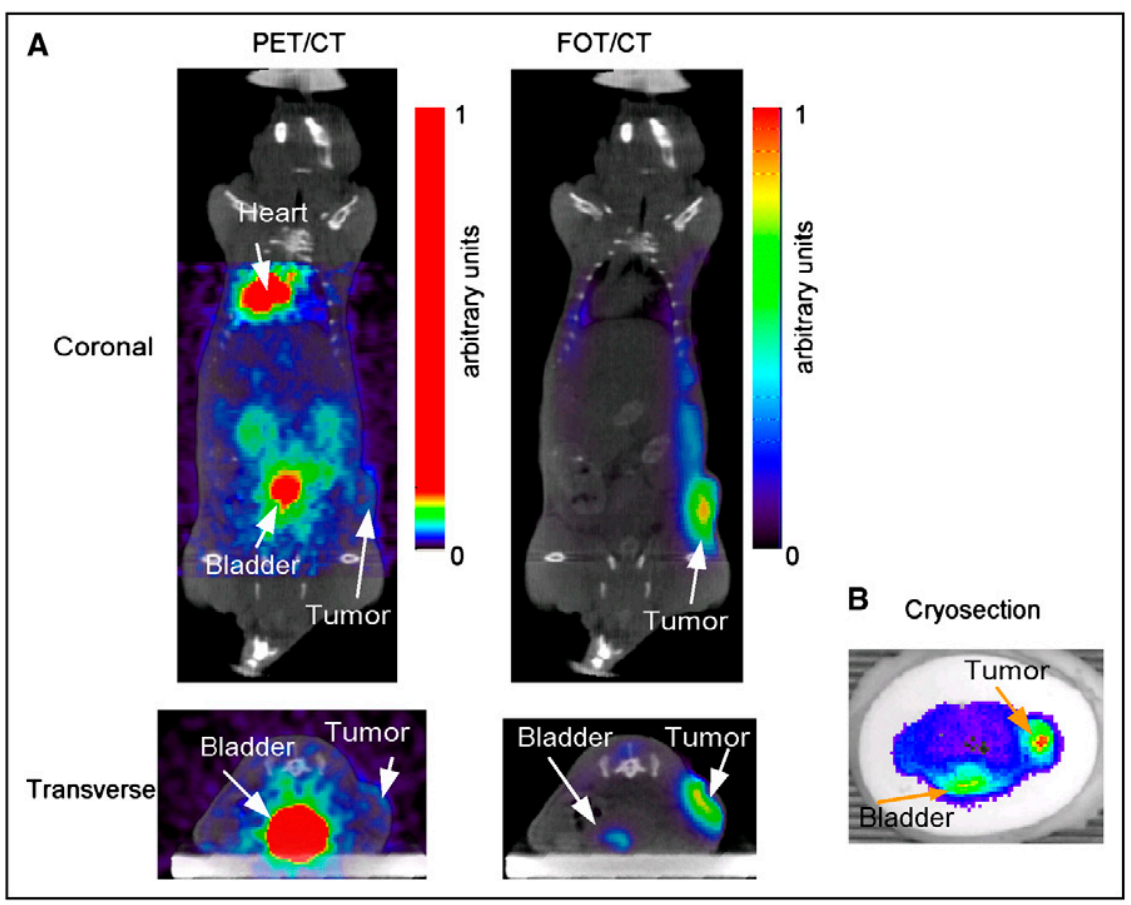


The in vivo study provides interesting information on differences in the biodistribution and tumor uptake of ${ }^{18} \mathrm{~F}-$ FDG, compared with IRDye $800 \mathrm{CW}$ conjugated with 2-DG. 3D FOT reconstruction results were supported by the distribution measured from ex vivo cryosection data. One hypothesis for the higher uptake of 2-DG, compared with ${ }^{18} \mathrm{~F}-\mathrm{FDG}$, in this particular model is that the tumor was quite small and appears to have been poorly perfused. Because 2-DG is cleared more slowly and has a much longer circulation time, the concentration profile presented to the tumor is higher than with ${ }^{18} \mathrm{~F}-\mathrm{FDG}$, possibly overcoming perfusion limitations. Alternatively, the uptake mechanisms for the 2 probes may differ, and the differences observed may reflect a preference for one mechanism over the other in this particular cell line. These results highlight the need for multimodal imaging systems and experiments that can further study such questions.

\section{CONCLUSION}

We developed a simultaneous PET and 3D FOT imaging system for small animals based on a novel truncated conical mirror insert and evaluated system performance. Phantom and in vivo experiments demonstrated the feasibility of simultaneous dual-modality imaging. Future plans involve developing a new mirror to reduce photon attenuation and scatter in PET, extension to multispectral FOT, and the use of a multimodal phantom to compute the rigid registration of the PET and FOT image volumes and account for any small variability in mirror positioning within the PET scanner. Finally, we plan to use this system to study multimodality imaging probes and complementary pairs of radiotracers and fluorescent probes in vivo.

\section{DISCLOSURE STATEMENT}

The costs of publication of this article were defrayed in part by the payment of page charges. Therefore, and solely to indicate this fact, this article is hereby marked "advertisement" in accordance with 18 USC section 1734.

\section{ACKNOWLEDGMENTS}

We thank the Center for Molecular and Genomic Imaging for assistance with animal studies, and Dr. Julie Sutcliffe and Dr. Sven Hausner for the xenograft model. We also thank Drs. Richard Leahy, Sangtae Ahn, and Joyita Dutta at the University of Southern California for useful discussions. This work was funded by NIH grant R01 CA121783, DOE grant DE-SC0002294, and an SNM Postdoctoral Molecular Imaging Scholarship. No other potential conflict of interest relevant to this article was reported.

\section{REFERENCES}

1. Cherry SR. Multimodality in vivo imaging systems: twice the power or double the trouble? Annu Rev Biomed Eng. 2006;8:35-62.

2. Townsend DW. Multimodality imaging of structure and function. Phys Med Biol. 2008;53:R1-R39.

3. Ale A, Schulz RB, Sarantopoulos A, Ntziachristos V. Imaging performance of a hybrid x-ray computed tomography-fluorescence molecular tomography system using priors. Med Phys. 2010;37:1976-1986.

4. Culver J, Akers W, Achilefu S. Multimodality molecular imaging with combined optical and SPECT/PET modalities. J Nucl Med. 2008;49:169-172.

5. Ray P, De A, Min JJ, Tsien RY, Gambhir SS. Imaging tri-fusion multimodality reporter gene expression in living subjects. Cancer Res. 2004;64:1323-1330.

6. Houston JP, Ke S, Wang W, Li C, Sevick-Muraca EM. Quality analysis of in vivo near-infrared fluorescence and conventional gamma images acquired using a dual-labeled tumor-targeting probe. J Biomed Opt. 2005;10:054010.

7. Zhang Z, Liang KX, Bloch S, Berezin M, Achilefu S. Monomolecular multimodal fluorescence-radioisotope imaging agents. Bioconjug Chem. 2005;16:1232-1239.

8. Sampath L, Kwon S, Ke S, et al. Dual-labeled trastuzumab-based imaging agent for the detection of human epidermal growth factor receptor 2 overexpression in breast cancer. J Nucl Med. 2007;48:1501-1510.

9. Rowland DJ, Cherry SR. Small-animal preclinical nuclear medicine instrumentation and methodology. Semin Nucl Med. 2008;38:209-222.

10. Culver JP, Choe R, Holboke MJ, et al. Three-dimensional diffuse optical tomography in the parallel plane transmission geometry: evaluation of a hybrid frequency domain/continuous wave clinical system for breast imaging. Med Phys. 2003;30:235-247.

11. Garofalakis A, Zacharakis G, Meyer H, et al. Three-dimensional in vivo imaging of green fluorescent protein-expressing $\mathrm{T}$ cells in mice with noncontact fluorescence molecular tomography. Mol Imaging. 2007;6:96-107.

12. Ntziachristos V, Ripoll J, Wang LHV, Weissleder R. Looking and listening to light: the evolution of whole-body photonic imaging. Nat Biotechnol. 2005;23: 313-320.

13. Zacharakis G, Ripoll J, Weissleder R, Ntziachristos V. Fluorescent protein tomography scanner for small animal imaging. IEEE Trans Med Imaging. 2005;24: 878-885.

14. Funovics MA, Weissleder R, Mahmood U. Catheter-based in vivo imaging of enzyme activity and gene expression: feasibility study in mice. Radiology. 2004; 231:659-666.

15. Li C, Wang G, Qi J, Cherry SR. Three-dimensional fluorescence optical tomography in small-animal imaging using simultaneous positron-emission-tomography priors. Opt Lett. 2009;34:2933-2935.

16. Gambhir SS, Herschman HR, Cherry SR, et al. Imaging transgene expression with radionuclide imaging technologies. Neoplasia. 2000;2:118-138.

17. Peter J, Unholtz D, Schulz RB, Doll J, Semmler W. Development and initial results of a tomographic dual-modality positron/optical small animal imager. IEEE Trans Nucl Sci. 2007;54:1553-1560.

18. Jung JH, Choi Y, Hong KJ, et al. Development of a dual modality imaging system: a combined gamma camera and optical imager. Phys Med Biol. 2009;54:4547-4559.

19. Alexandrakis G, Rannou FR, Chatziioannou AF. Tomographic bioluminescence imaging by use of a combined optical-PET (OPET) system: a computer simulation feasibility study. Phys Med Biol. 2005;50:4225-4241.

20. Li C, Mitchell GS, Dutta J, Ahn S, Leahy RM, Cherry SR. A three-dimensional multispectral fluorescence optical tomography imaging system for small animals based on a conical mirror design. Opt Express. 2009;17:7571-7585.

21. Tai YC, Chatziioannou AF, Yang YF, et al. MicroPET II: design, development and initial performance of an improved microPET scanner for small-animal imaging. Phys Med Biol. 2003;48:1519-1537.

22. Yang Y, Tai YC, Siegel S, et al. Optimization and performance evaluation of the microPET II scanner for in vivo small-animal imaging. Phys Med Biol. 2004;49: 2527-2545.

23. Kovar JL, Volcheck W, Sevick-Muraca E, Simpson MA, Olive DM. Characterization and performance of a near-infrared 2-deoxyglucose optical imaging agent for mouse cancer models. Anal Biochem. 2009;384:254-262.

24. Qi J, Leahy RM, Cherry SR, Chatziioannou A, Farquhar TH. High-resolution 3D Bayesian image reconstruction using the microPET small-animal scanner. Phys Med Biol. 1998;43:1001-1013. 